\title{
Resolving electron transfer kinetics at the nanocrystal/solution interface
}

\author{
Peter Liljeroth $^{\dagger}$, Bernadette M. Quinn ${ }^{{ }^{*}}$ \\ ${ }^{\dagger}$ Condensed Matter and Interfaces, Debye Institute, University of Utrecht, \\ PO Box 80000, 3508 TA Utrecht, the Netherlands \\ ${ }^{\ddagger}$ Laboratory of Physical Chemistry and Electrochemistry, Helsinki \\ University of Technology, PO Box 6100, 02015 HUT Finland \\ *Corresponding Author. Email: bquinn@iki.fi
}

\section{SUPPORTING INFORMATION}

\section{Additional experimental details}

Nanoparticle synthesis

The nanoparticle synthesis has been described in detail in a previous publication. Briefly, dodecanethiolate-protected gold particles with sub- $2 \mathrm{~nm}$ core diameters were synthesized using the standard two-phase Brust-Schriffin method. ${ }^{\text {1 }}$ To increase core size and improve monodispersity, the particles were then annealed according to the method reported by Shimizu et al. ${ }^{\mathrm{S} 2}$ Crude dodecanethiol protected gold NCs were rotavapped to dryness without removal of the excess tetraoctylammonium salts, and the resulting black solid was heat-treated at $190{ }^{\circ} \mathrm{C}$ for $30 \mathrm{~min}$ in a furnace at a heating rate of $2{ }^{\circ} \mathrm{C} / \mathrm{min}$. After heat treatment, the product was cleaned by dissolution/precipitation cycles. Evolution of core size was confirmed by both the appearance of a surface plasmon in the UV spectra and TEM images of drop cast films on carbon-coated copper grids. The observed particle diameter was $6.6 \pm 0.8 \mathrm{~nm}$.

\section{Deposition of the nanocrystal monolayers}

Rendering the glass substrates hydrophobic (silanization treatment) enabled us to obtain high-quality Langmuir-Schaefer deposition. This is supported by absorbance measurements of the films: the optical properties of a Au nanocrystal monolayer depend on the interparticle separation. ${ }^{\mathrm{S} 3}$ The surface plasmon resonance red-shifts due to the dipolar coupling between the particles. The optical properties of the film were unchanged upon the transfer from the water-air interface onto a glass slide. In addition, TEM imaging of films transferred onto TEM grids showed high-quality monolayers. Finally, the observation of an insulator to metal transition in the transferred nanocrystal monolayers as the deposition surface pressure was increased lends support to highfidelity transfer. ${ }^{\mathrm{S} 3}$ 


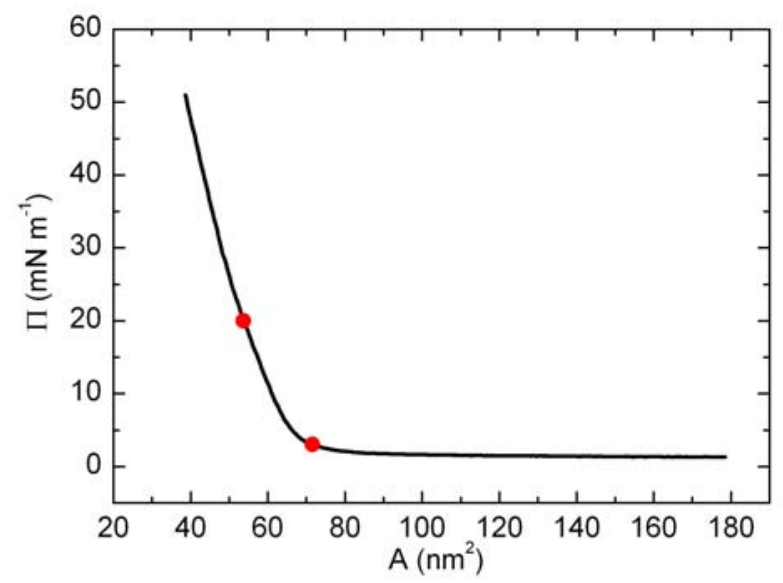

Figure S1. Surface pressure as a function of the area per nanocrystal measured in a Langmuir trough. The monolayers used for the SECM experiments were deposited by the Langmuir-Schaefer method at the surface pressures of 3 and $20 \mathrm{mN} \mathrm{m}^{-1}$ (red symbols).

\section{Strategy of the SECM experiments}

Low concentrations of redox mediator are required for the SECM measurements for the insulating $\mathrm{NC}$ film (transferred at $3 \mathrm{mN} \mathrm{m}^{-1}$ ). The experimental protocol for recording approach curves at low concentrations is detailed here: initial measurements were performed using the highest concentration of $\mathrm{Fe}(\mathrm{CN})_{6}{ }^{4-}$. Cyclic voltammograms were recorded in bulk solution, far form the substrate. The tip was then biased at the diffusionlimited oxidation potential for $\mathrm{Fe}(\mathrm{CN})_{6}{ }^{4-}$ and an approach curve was recorded. The tip was then retracted (typically $50 \mu \mathrm{m}$ ) and the solution (a drop of solution on the silanized glass slide onto which the NC monolayer had been transferred) was changed by removing it with a pipette and pipetting a fresh solution with a different concentration of $\mathrm{Fe}(\mathrm{CN})_{6}{ }^{4-}$. An approach curve for this concentration of mediator was then measured, followed again by tip retraction. This was repeated for several concentrations of redox mediator (constant base electrolyte concentration). In this manner, it was possible to a) always probe the same spot (several spots were tested and responses obtained were practically identical) and b) obtain the distance of the tip from the substrate with respect to the previously measured concentration. An example of the repeatability of the measurements is shown in Figure S2. The slight oscillations in the approach curves were present in the background measurement and are hence the same in both curves. 


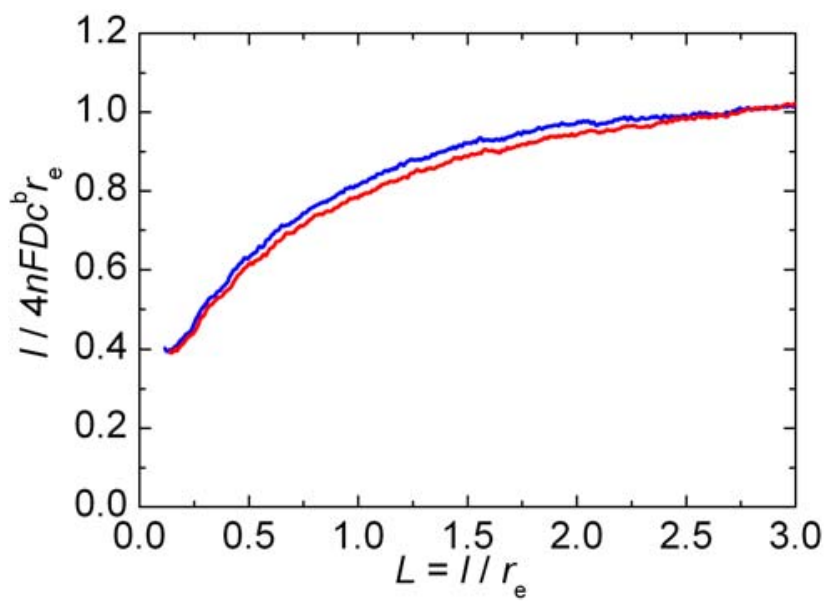

Figure S2. Experimental approach curves recorded to the NC film transferred at $3 \mathrm{mN}$ $\mathrm{m}^{-1}$ at the low concentration of $\mathrm{Fe}(\mathrm{CN})_{6}{ }^{4-}: 2.3$ (blue line) and $2.5 \mu \mathrm{M}$ (red line) illustrating the repeatability of the SECM experiments.

The background approach curve was measured by exchanging the solution with one containing base electrolyte only, and repeating the experiment (applying the same potential to the tip electrode). This is shown in Figure S3. In this way, spurious results due to these low concentrations of mediator were discounted. Experimental and background corrected approach curves are given in Figure S3.



Figure S3. Experimental approach curves recorded to the NC film transferred at $3 \mathrm{mN} \mathrm{m}^{-}$ ${ }^{1}$ at the lowest concentrations of $\mathrm{Fe}(\mathrm{CN})_{6}{ }^{4-}$ considered 1.1 (solid blue line) and $5.6 \mu \mathrm{M}$ (solid red line). The black line shows the approach curve obtained in the complete absence of the redox mediator and gives the background current at the Pt SECM electrode. The plot shows the experimental approach curves pre- (solid lines) and post(dotted lines) subtraction of the background approach curve (black line).

\section{Details of the SECM modeling}

The theoretical treatment has been discussed in detail in an earlier publication. ${ }^{\mathrm{S} 3} \mathrm{We}$ repeat here the salient features of the model and, in particular focus on the effect of the 
kinetics of the electron transfer reaction between the solution redox couple and the nanocrystals on the feedback response.

The theoretical analysis shown here follows a standard treatment of SECM masstransport problem. ${ }^{\text {S4-6 }}$ The problem of lateral transport on the substrate has been analyzed in earlier publications, ${ }^{\text {S7-12 }}$ and the present work gives an extension to Ohmic behavior on the substrate. The electrochemical potential of electrons in the NC monolayer, $\mu$, is given by

$$
\mu=\mu^{0}-e\left(\phi(r)-\phi^{0}\right)
$$

where $\mu^{0}$ is the standard electrochemical potential (corresponding to the electrostatic potential $\left.\phi^{0}\right)$, $e$ the elementary charge, $\phi(r)$ the local electrostatic potential in the film and $r$ the radial coordinate. The equilibrium electrochemical potential in the film, $\mu_{\text {eq }}$, is set by the concentration ratio of the redox couple in solution

$$
\mu_{\mathrm{eq}}=\mu^{0}+k_{\mathrm{B}} T \ln \left(c_{\mathrm{Red}}^{\mathrm{b}} / c_{\mathrm{Ox}}^{\mathrm{b}}\right)
$$

where $c_{\mathrm{Red}}^{\mathrm{b}}$ and $c_{\mathrm{Ox}}^{\mathrm{b}}$ are the bulk concentrations of the reduced and oxidized forms of the redox couple, respectively, $k_{\mathrm{B}}$ the Boltzmann constant and $T$ the absolute temperature. Following conventional Gerischer theory of electron transfer, the rate constants of the reduction (forward reaction), $k_{\mathrm{f}}$, and oxidation (reverse reaction), $k_{\mathrm{b}}$, reactions between the solution redox couple and the nanoparticle monolayer can be expressed as follows

$$
\begin{aligned}
k_{\mathrm{f}} & =k^{0}\left(\frac{c_{\mathrm{Red}}^{\mathrm{b}}}{c_{\mathrm{Ox}}^{\mathrm{b}}}\right)^{1 / 2} \exp \left(\frac{\mu-\mu_{\mathrm{eq}}}{2 k T}\right) \\
k_{\mathrm{b}} & =k^{0}\left(\frac{c_{\mathrm{Ox}}^{\mathrm{b}}}{c_{\mathrm{Red}}^{\mathrm{b}}}\right)^{1 / 2} \exp \left(-\frac{\mu-\mu_{\mathrm{eq}}}{2 k T}\right)
\end{aligned}
$$

where

$$
k^{0}=\frac{k T \Gamma T\left(\mu_{e q}\right) g\left(\mu_{e q}\right)}{\left(4 \pi k_{\mathrm{B}} T \lambda\right)^{1 / 2}} \exp (-\lambda / 4 k T)
$$

where $T\left(\mu_{e q}\right)$ is the transmission coefficient at equilibrium, $g\left(\mu_{e q}\right)$ the density of states of the metal and $\lambda$ the reorganization energy. We use the local form of Ohm's law

$$
i=-\sigma \frac{\partial \phi}{\partial r}
$$

where $i$ is the current density and $\sigma$ is the conductivity, and introduce the following dimensionless quantities

$$
\begin{gathered}
\tilde{\mu}=\frac{\mu-\mu^{0}}{k T} \\
C=\frac{c_{\mathrm{Red}}}{c_{\mathrm{Red}}^{\mathrm{b}}+c_{\mathrm{Ox}}^{\mathrm{b}}}=\frac{c_{\mathrm{Red}}}{c^{\mathrm{b}}}
\end{gathered}
$$




$$
\begin{gathered}
R=r / r_{\mathrm{e}} \\
K^{0}=k^{0} r_{e} / D \\
\Sigma=\frac{\sigma k_{\mathrm{B}} T \Delta z}{e^{2} r_{\mathrm{e}} D c^{\mathrm{b}} N_{\mathrm{A}}}
\end{gathered}
$$

where $r_{\mathrm{e}}$ is the microelectrode radius, $D$ the diffusion coefficient of the solution redox couple, and $\Delta z$ the thickness of the monolayer. We then obtain Eq. (1) of the manuscript

$$
\frac{\partial^{2} \tilde{\mu}}{\partial R^{2}}+\frac{1}{R} \frac{\partial \tilde{\mu}}{\partial R}-\frac{K^{0}}{\Sigma}\left((1-C) e^{\tilde{\mu} / 2}-C e^{-\tilde{\mu} / 2}\right)=0
$$

The boundary conditions for the diffusion equation on the substrate are

$$
\begin{gathered}
\left.\frac{\partial \tilde{\mu}}{\partial R}\right|_{R=0}=0 \\
\tilde{\mu}(R \rightarrow \infty)=\tilde{\mu}_{\mathrm{eq}}
\end{gathered}
$$

where $\tilde{\mu}_{\mathrm{eq}}$ is the equilibrium electrochemical potential of the film (with respect to standard chemical potential of the redox couple). Note that even if only one form the redox couple is added, the equilibrium potential in reality is not $\pm \infty$. There is always a trace of the other redox species present (due to reactions with, for example, water or oxygen) and this sets the equilibrium potential.

In order to couple Eq. S12 to the diffusion problem in solution, the following boundary condition for the electron transfer is used

$$
\left.\frac{\partial C}{\partial Z}\right|_{Z=L}=K^{0}\left((1-C) e^{\tilde{\mu} / 2}-C e^{-\tilde{\mu} / 2}\right)
$$

where $L=l / a$ is the dimensionless tip-substrate distance. In the numerical solution, the effect of so-called back-diffusion, i.e. diffusion from behind the plane of the microelectrode, was included. The SECM tip is characterized by the radius of the insulating glass sheath, $R_{\mathrm{G}}=r_{\mathrm{G}} / r_{\mathrm{e}}$, which is determined from calibration measurements to insulating and conducting substrates. For details of the procedure, see for example the supporting information of reference S12. The diffusion problem was eventually solved for various values of $L$ with a commercial finite element method package (FEMLAB, Comsol Ab, Sweden) for given values of $R_{\mathrm{G}}, \Sigma, K^{0}$ and $\tilde{\mu}_{\mathrm{eq}}$ in order to extract SECM approach curves (current normalized by the current far from the substrate as a function of the tip-substrate separation, $L$ ). The finite element mesh was first refined adaptively with $\Sigma=0$ and then the full, non-linear, problem was solved with that mesh. The number of elements employed was ordinarily ca. $5 \times 10^{4}$. 

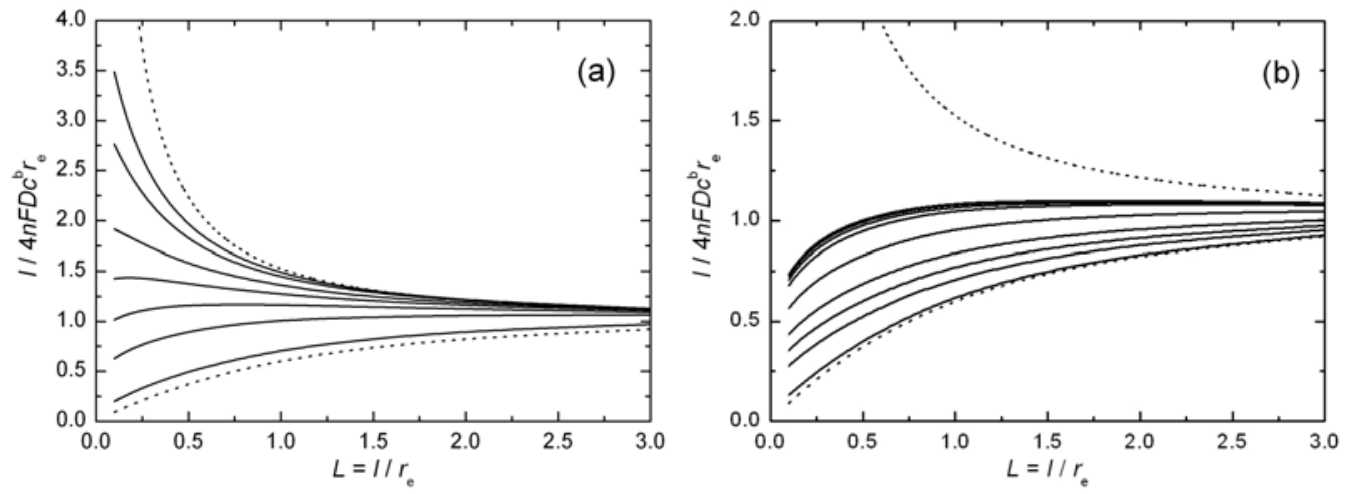

Figure S4. (a) Simulated approach curves for $\Sigma=2.5$ and $K^{0}=100,3,1,0.5,0.25,0.1$ and 0.01 (top to bottom). (b) Simulated approach curves for $K^{0}=0.1$ and $\Sigma=100,65,23$, $11,5.5,1.3,0.43,0.21,0.1$ and 0.01 (top to bottom). The upper and lower dashed lines are the theoretical responses for positive and negative feedback, respectively. $C=0.96$ corresponding to $\tilde{\mu}_{\mathrm{eq}}=3.17\left(E_{\mathrm{eq}}=-82 \mathrm{mV}\right.$ with respect to the formal potential of the redox couple)

A series of simulated approach curves are shown in Figure S4. Figure S4a demonstrates the effect of varying the standard rate constant for a given value of the dimensionless conductivity. It can be seen that the response can vary between negative and positive feedback depending on the kinetics. Figure S4b show the effect of varying the dimensionless conductivity, where the feedback response saturates to given value determined by $K^{0}$ (and $\tilde{\mu}_{\mathrm{eq}}$ ). In the experiments, for a given film conductivity, lowering the concentration of the solution redox couple will increase the dimensionless conductivity $\left(\Sigma \propto \sigma / c^{b}\right)$ and if sufficiently low concentrations can be used, behavior shown in Figure S4b should be seen. The more negative the equilibrium potential of the film is made ( $\tilde{\mu}_{\mathrm{eq}} \rightarrow-\infty, c_{\mathrm{Ox}}^{\mathrm{b}} \rightarrow 0$ ), the more positive feedback is observed.

\section{Comparison of conductivity determined with $\mathrm{FcMeOH}$ and $\mathrm{Fe}(\mathrm{CN})_{6}{ }^{4-}$}

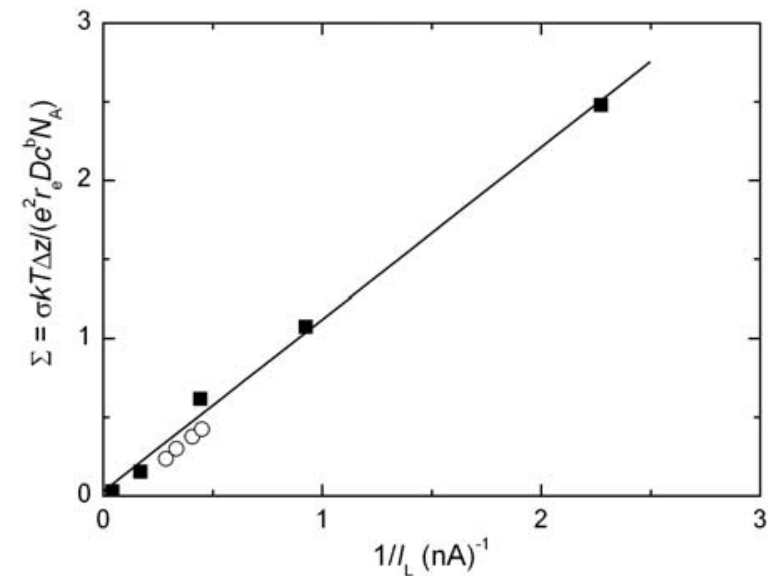

Figure S5. Plot of the dimensionless conductivity as a function of the reciprocal tip limiting current far from the substrate for $\mathrm{Fe}(\mathrm{CN})_{6}{ }^{4-}$ (squares) and $\mathrm{FcMeOH}$ (circles). 


\section{SECM approach curves with the NC monolayer deposited at $3 \mathrm{mN} \mathrm{m}^{-1}$}

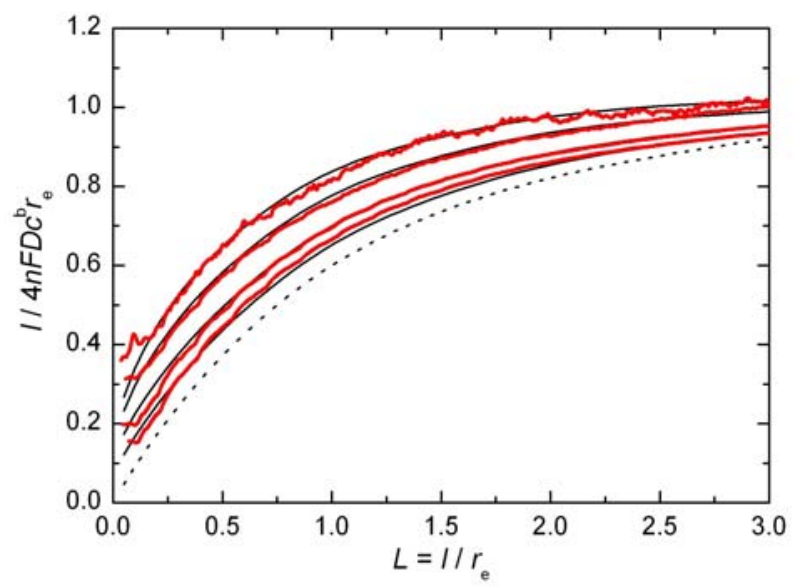

Figure S6. Experimental approach curves recorded to the NC film transferred at $3 \mathrm{mN}$ $\mathrm{m}^{-1}$ (red) for a range of $\mathrm{Fe}(\mathrm{CN})_{6}{ }^{4-}$ concentrations (top to bottom, 1.1, 5.6, 27, and $96 \mu \mathrm{M}$ ) and the corresponding fits to the theory (black) $(\Sigma=3.61,0.72,0.15,0.04$ top to bottom for $\left.K^{0}=0.03, \tilde{\mu}_{e q}=3.17\right)$. The dashed line is the theoretical response for negative feedback.

\section{Full citation for reference (1) in the communication}

Adams, D. M.; Brus, L.; Chidsey, C. E. D.; Creager, S.; Creutz, C.; Kagan, C. R.; Kamat, P. V.; Lieberman, M.; Lindsay, S.; Marcus, R. A.; Metzger, R. M.; Michel-Beyerle, M. E.; Miller, J. R.; Newton, M. D.; Rolison, D. R.; Sankey, O.; Schanze, K. S.; Yardley, J.; Zhu, X. J. Phys. Chem. B 2003, 107, 6668.

\section{References}

(S1) Brust, M.; Walker, M.; Bethell, D.; Schiffrin, D. J.; Whyman, R. J. Chem. Soc.,Chem. Commun. 1994, 801.

(S2) Shimizu, T.; Teranishi, T.; Hasegawa, S.; Miyake, M. J. Phys. Chem. B 2003, 107, 2719.

(S3) Liljeroth, P.; Vanmaekelbergh, D.; Ruiz, R.; Kontturi, K.; Jiang, H.; Kauppinen, E.; Quinn, B. M. J. Am. Chem. Soc. 2004, 126, 7126.

(S4) Bard, A. J.; Mirkin, M. V.; Unwin, P. R.; Wipf, D. O. J. Phys. Chem. 1992, 96, 1861.

(S5) Bard, A. J.; Fan, F. R. F.; Mirkin, M. V. In Electroanalytical Chemistry; Bard, A. J., Ed.; Marcel Dekker: New York, 1994; Vol. 18, pp 243.

(S6) Bard, A. J.; Faulkner, L. R. Electrochemical methods, Fundamentals and applications; 2nd ed.; John Wiley \& Sons Inc.: New York, 2001.

(S7) Unwin, P. R.; Bard, A. J. J. Phys. Chem. 1992, 96, 5035.

(S8) Slevin, C. J.; Unwin, P. R. J. Am. Chem. Soc. 2000, 122, 2597.

(S9) Zhang, J.; Slevin, C. J.; Morton, C.; Scott, P.; Walton, D. J.; Unwin, P. R. J. Phys. Chem. B 2001, $105,11120$.

(S10) Mandler, D.; Unwin, P. R. J. Phys. Chem. B 2003, 107, 407.

(S11) Liljeroth, P.; Quinn, B. M.; Ruiz, V.; Kontturi, K. Chem. Commun. 2003, 1570.

(S12) Ruiz, V.; Liljeroth, P.; Quinn, B. M.; Kontturi, K. Nano Lett. 2003, 3, 1459. 